\title{
Extended Wiener Measure by Nonstandard Analysis for Financial Time Series
}

\author{
Shuya Kanagawa', Ryoukichi Nishiyama ${ }^{2}$, Kiyoyuki Tchizawa ${ }^{3}$ \\ ${ }^{1}$ Epartment of Mathematics, Tokyo City University, Tokyo, Japan \\ ${ }^{2}$ Department of literature, Ryukoku University, Kyoto, Japan \\ ${ }^{3}$ Institute of Administration Engineering, Ltd., Tokyo, Japan \\ Email: skanagaw@tcu.ac.jp, rynish@y6.dion.ne.jp, tchizawa@kthree.co.jp
}

How to cite this paper: Kanagawa, S., Nishiyama, R. and Tchizawa, K. (2018) Extended Wiener Measure by Nonstandard Analysis for Financial Time Series. Applied Mathematics, 9, 975-984. https://doi.org/10.4236/am.2018.98066

Received: June 22, 2018

Accepted: August 27, 2018

Published: August 30, 2018

Copyright $\odot 2018$ by authors and Scientific Research Publishing Inc. This work is licensed under the Creative Commons Attribution International License (CC BY 4.0).

http://creativecommons.org/licenses/by/4.0/

c) (i) Open Access

\begin{abstract}
We propose a new approach to construct an extended Wiener measure using nonstandard analysis by E. Nelson. For the new definition we construct non-standardized convolution of probability measure for independent random variables. As an application, we consider a simple calculation of financial time series.
\end{abstract}

\section{Keywords}

Time Series, Black-Sholes Model, S-Continuity, Nonstandard Analysis, Delta-Function

\section{Introduction}

As for option pricing in financial mathematics we investigate the expectation of a functional of the Wiener process which can be represented as the integral with respect to the Wiener measure. Unfortunately it is difficult to calculate the Wiener integral using standard methods directly. Therefore Black and Scholes [1] investigated the PDE (5) for the option pricing. Then we choose another extended method, i.e. nonstandard analysis, to calculate the Wiener integral. It links the Wiener measure to the Black-Sholes model, which is of the Heat equations. It is known that their solutions are constructed by the Bessel's special functions. As they are described by the Fourier integral, we need to use the convolution of the non-standard version. We give some sufficient conditions to make it possible to this procedure. In 1988, M. Kionoshita introduced non-standard analysis for distributions [2]. It is one of the former investigations. 


\section{Construction of Extended Wiener Measure}

\subsection{Definition of Standard Wiener Measure}

Wiener measure $\mathbf{W}$ on $C[0, T]$ is defined by

$$
\begin{aligned}
& \mathbf{W}\left\{\omega \in C[0, T] \mid \omega\left(t_{1}\right) \in A_{1}, \cdots, \omega\left(t_{k}\right) \in A_{k}\right\} \\
& =\int_{A_{1}} \cdots \int_{A_{k}} p\left(t_{1}, x_{1}\right) p\left(t_{2}-t_{1}, x_{2}-x_{1}\right) \cdots p\left(t_{k}-t_{k-1}, x_{k}-x_{k-1}\right) \mathrm{d} x_{1} \cdots \mathrm{d} x_{k}
\end{aligned}
$$

for any $0 \leq t_{1} \leq \cdots \leq t_{n} \leq T$ and any Borel sets $A_{1}, \cdots, A_{k}$, where

$$
p(t, x)=\frac{1}{\sqrt{2 \pi t}} \mathrm{e}^{-\frac{x^{2}}{2 t}}
$$

\subsection{Nonstandard Convolution by Hyper Function}

Let $\mathcal{F}$ be the space of all locally integrable functions on $\mathbf{R}$. Define the space of all rapidly decreasing $C^{\infty}$ functions $\mathcal{S}$ by

$$
\sup _{x \in R}\left|x^{m} f^{(k)}(x)\right|<\infty
$$

for any $m, k \in \mathbf{Z}_{+}$. Furthermore define the space of all slowly increasing functions $\mathcal{O}$ by for any $k \in \mathbf{Z}_{+}$there exist some positive constants $C_{k}$ and $M_{k} \in \mathbf{Z}_{+}$such that

$$
\left|f^{(k)}(x)\right|<C_{k}(1+|x|)^{M_{k}} .
$$

$f \in \mathcal{F}$ is Lebesgue integrable on any compact set $K$ such that

$$
-\infty<\int_{K} f(x) \mathrm{d} x<\infty
$$

For $f \in C^{\infty}$ there exists continuous $k$-th derivative $f^{(k)}(x)$ for each $k \geq 1$. For $f \in S \subset C^{\infty}$

$$
\sup _{x \in R}\left|x^{m} f^{(k)}(x)\right|<\infty
$$

holds for each $k, m \in Z_{+}$, where $Z_{+}$means the space of all positive integers.

From the above we can easily obtain that

$$
S \subset \mathcal{O} \subset C^{\infty} \subset F
$$

For $f, g \in S$, the convolution of $f$ and $g$ is defined by

$$
f * g(x)=\int_{-\infty}^{\infty} f(s) g(x-s) \mathrm{d} s
$$

The definition of the convolution can be extended to the $k$-th convolution by

$$
f_{1} * f_{2} * \cdots * f_{k}(x)=\int_{-\infty}^{\infty}\left(f_{1} * \cdots * f_{k-1}\right)(s) f_{k}(x-s) \mathrm{d} s
$$

for each $k \in Z_{+}$.

From the above definitions we can easily obtain the next result.

Proposition 1.

1) $f * g(x)=g * f(x)$.

2) $f *(g+h)(x)=g * f(x)+g * h(x)$. 
3) $\operatorname{supp}(f * g) \subseteq \operatorname{supp}(f) \bigcup \operatorname{supp}(g)$.

Proposition 2. For $\varphi, \psi \in S$

1) $\varphi \psi \in S$.

2) $\varphi * \psi \in S$.

3) $(\varphi * \psi)^{(k)}(x)=\varphi^{(k)} * \psi(x)=\varphi * \psi^{(k)}(x)$.

Let $\tilde{H}$ be the set of rapidly decreasing functions satisfying

1) $f(-x)=f(x)$.

2) $\int_{-\infty}^{\infty} f(x) \mathrm{d} x=1$

Define a function $\bar{f}(t)$ by

$$
\bar{f}(t)(x)=\frac{1}{t} f\left(\frac{x}{t}\right), t \in(0,1]
$$

\section{Proposition 3.}

1) $f \in \tilde{H} \Rightarrow \bar{f}(t) \in \tilde{H}$.

2) $f, g \in \tilde{H} \Rightarrow f * g \in \tilde{H}$.

\section{Proof.}

1) can be shown easily. We next prove 2). Since $f * g \in S$ from Proposition 2 2), we have

1)

$$
\begin{aligned}
f * g(-x) & =\int_{-\infty}^{\infty} f(s) g(-x-s) \mathrm{d} s=\int_{-\infty}^{\infty} f(-s) g(x+s) \mathrm{d} s \\
& =\int_{\infty}^{-\infty} f(u) g(x-u)(-\mathrm{d} u)=\int_{-\infty}^{\infty} f(u) g(x-u) \mathrm{d} u=f * g(x)
\end{aligned}
$$

2)

$$
\begin{aligned}
\int_{-\infty}^{\infty} f * g(x) \mathrm{d} x & =\int_{-\infty}^{\infty} \int_{-\infty}^{\infty} f(s) g(x-s) \mathrm{d} s \mathrm{~d} x \\
& =\int_{-\infty}^{\infty} \int_{-\infty}^{\infty} f(u) g(v) \mathrm{d} u \mathrm{~d} v=\int_{-\infty}^{\infty} f(u) \mathrm{d} u \int_{-\infty}^{\infty} g(v) \mathrm{d} v=1
\end{aligned}
$$

Remark 1. If a distribution $U$ is a linear form and continuous on $S$, then $U$ is called slowly increasing.

The Dirac distribution $\delta$ is defined by

$$
\delta(\phi)=\phi(0), \phi \in S
$$

Let $V$ be a set of the slowly increasing distributions and let $L_{f} \in V$ be defined by

$$
L_{f}(\varphi)=\langle f, \phi\rangle, \phi \in S .
$$

Then $\langle f, \phi\rangle$ is the inner product and it is well defined when

$$
\langle f, \phi\rangle=\int_{-\infty}^{\infty} f(x) \phi(x) \mathrm{d} x
$$

is integrable.

Let ${ }^{*} R$ be the set of hyper real numbers. The hyper function $f_{\delta}:{ }^{*} R \rightarrow{ }^{*} R$ is defined by

$$
f_{\delta}\left(a_{\delta}\right)=b_{\delta}, \quad b(t)=f_{t}(a(t)), \quad t \in(0,1]
$$

for a representative $a(t)$ of $a_{\delta} \in{ }^{*} R$, where $\left\{f_{t}(\cdot) \mid t \in(0,1]\right\}$ is a family of 
functions. Let ${ }^{*} \mathcal{F},{ }^{*} \mathrm{C}^{\infty},{ }^{*} \mathcal{S}$ and ${ }^{*} \mathcal{O}$ be the set of hyper integrable functions, the set of hyper $\mathcal{C}^{\infty}$ functions, the set of slowly decreasing hyper $\mathcal{C}^{\infty}$ functions and the set of slowly increasing hyper $\mathcal{C}^{\infty}$ functions, respectively.

In the above definitions, "it is a prediction $P(t)$ " is equivalent to " $P(t)$ for any $t \in J, J \in \mathcal{U}$ on $(0,1]$, where $\mathcal{U}$ is an $\omega$-incomplete ultra filter, is prediction". In other words, at each neighborhood of the zero on $(0,1] P(t)$ is established almost everywhere.

Next we define the hyper inner product by

$$
{ }^{*}\left\langle f_{\delta}, g_{\delta}\right\rangle=a_{\delta} \in{ }^{*} R, \quad f_{\delta}, g_{\delta} \in \mathcal{F}, \quad a(t)=\langle f(t), g(t)\rangle \in R(t \in(0,1])
$$

Furthermore the convolution of hyper functions is defined by

$$
f_{\delta} * g_{\delta}=h_{\delta}, \quad h(t)=f(t) * g(t) \quad(t \in(0,1])
$$

For the convolution of hyper functions, analogous results of Propositions 1 and 2 hold.

The hyper operator ${ }^{*} T$ is also defined by

$$
{ }^{*} T\left(\phi_{s}\right)=a_{\delta} \in{ }^{*} R, \quad a(t)=T\left(\phi_{s}\right) \quad \text { for } T \in \mathcal{T}, \phi_{s} \in{ }^{*} \mathcal{S}
$$

Remark 2. Similarly to the definition of the convolution in hyper functions, we can well define the convolution of hyper functions and hyper distributions, the convolution of hyper distributions and hyper functions and the convolution in distributions, respectively.

\subsection{Extended Wiener Measure}

Put

$$
t_{k}=\frac{T k}{\mathbf{N}}, k=0,1, \cdots, \mathbf{N}
$$

where $\mathbf{N}$ is a hyper natural number in the sense of nonstandard analysis.

Let $V$ be the space of simple random walks defined by

$$
V=\{\bar{\omega} \in C[0, T] \mid \bar{\omega} \text { is a simple random walk }\}
$$

where the simple random walk is defined by

$$
\begin{aligned}
& P\left\{\bar{\omega}\left(t_{k}\right)=x_{k-1}+1 / \sqrt{\mathbf{N}} \mid \bar{\omega}\left(t_{k-1}\right)=x_{k-1}\right\}=\frac{1}{2} \\
& P\left\{\bar{\omega}\left(t_{k}\right)=x_{k-1}-1 / \sqrt{\mathbf{N}} \mid \bar{\omega}\left(t_{k-1}\right)=x_{k-1}\right\}=\frac{1}{2}
\end{aligned}
$$

and

$$
\bar{\omega}(0)=x_{0}=0
$$

with probability 1 . Then $V$ is a support on the extended Wiener measure in $C[0, T]$. The fact is based on the central limit theorem.

For any set $A$ in the Borel $\sigma$-field $\mathfrak{B}$, the extended Wiener measure of $A$ is defined by 


$$
\mathbf{W}(A)=\frac{\#(A \cap V)}{2^{\mathbf{N}}}
$$

where $\#(A)$ the cardinality of $A \cap V$.

Theorem 2.1. For any Borel set $A \in \mathfrak{B}(C[0, T])$, the Wiener measure W can be represented by

$$
\mathbf{W}(A)=\sum_{\bar{\omega} \in A \cap V} \mathbf{W}(\bar{\omega})
$$

where $\mathfrak{B}(C[0, T])$ is the Borel field of the space of continuous functions on $[0, T]$.

Proof. Consider a calculus of the integral with respect to $W$ in the sense of nonstandard analysis. For $\bar{\omega}, \tilde{\omega} \in A \cap V$ with $\bar{\omega} \neq \tilde{\omega}$,

$$
\bar{\omega} \cap \tilde{\omega}=\varnothing
$$

Since $V$ is dense in $C[0, T]$, we have

$$
\bigcup_{\bar{\omega} \in A \cap V} \bar{\omega}=A
$$

Combining (2) and (3) we prove the theorem.

Theorem 2.2. For a measurable function $h$ on $C[0, T]$ we have

$$
\int_{C[0, T]} h(\omega) \mathbf{W}(\mathrm{d} \omega)=\sum_{\bar{\omega} \in V} h(\bar{\omega}) \mathbf{W}(\bar{\omega})
$$

\section{Black-Scholes Model}

Let $S(t), 0 \leq t \leq T$ be a stock price, where $T$ is a maturity. The well known Black-Scholes model [1], which describes the price evolution of some stock options, is the Itô stochastic differential equation

$$
\mathrm{d} S(t)=\mu S(t)+\sigma S(t) \mathrm{d} W(t)
$$

where

- $W(t)$ is a standard Wiener process,

- the volatility $\sigma$ and the $d r i f t$, or trend, $\mu$ are assumed to be constant.

This model and its numerous generalizations are playing a major role in financial mathematics since more than thirty years although Equation (4) is often severely criticized (see, e.g., [1] [3] and the references therein).

The solution of Equation (4) is the geometric Brownian motion which reads

$$
S(t)=S(0) \exp \left(\left(\mu-\frac{\sigma^{2}}{2}\right) t+\sigma W(t)\right)
$$

where $S(0)$ is the initial condition. It seems most natural to consider the mean $S(0) \mathrm{e}^{\mu t}$ of $S(t)$ as the trend of $S(t)$. This choice unfortunately does not agree with the following fact: $F(t)=S(t)-S(0) \mathrm{e}^{\mu t}$ is almost surely not a quickly fluctuating function around 0 , i.e., the probability that

$\left|\int_{0}^{T} F(\tau) s \mathrm{~d} \tau\right|>\epsilon>0, T>0$, is not "small", when

- $\epsilon$ is "small",

- $T$ is neither "small" nor "large". 
A rigorous treatment, which would agree with nonstandard analysis (see, e.g., [4] [5] [6]), may be deduced from some infinitesimal time-sampling of Equation (4).

\subsection{Option Pricing for One-Step Binomial Model}

Let $K$ be an exercise price. A derivative with a payoff

$$
D(T)=f(S(T))=\max \{S(T)-K, 0\}
$$

is called a call option. On the other hand a put option is a derivative with a payoff

$$
D(T)=f(S(T))=\max \{K-S(T), 0\} .
$$

We first consider the option premium by a one-step binomial tree model defined by

$$
S(T)=\left\{\begin{array}{l}
S(0)(1+u) \\
S(0)(1+d)
\end{array}\right.
$$

where $d \leq r \leq u$ are returns on the stock and $r$ is a risk-free rate. We next define a risk-neutral probability by

$$
\left\{\begin{array}{l}
p_{u}^{*}=\frac{r-d}{u-d} \\
p_{d}^{*}=\frac{u-r}{u-d}
\end{array}\right.
$$

and $E^{*}$ is the expectation under the risk-neutral probability $\left\{p_{u}^{*}, p_{d}^{*}\right\}$. Since the option premium can be obtained to discount the expectation of the payoff function $D(t)$, we have the following the call option premium $C_{0}$

$$
\begin{aligned}
C_{0} & =\frac{1}{1+r} E^{*}[D(T)] \\
& =\frac{1}{1+r}\left[p_{d}^{*} \max \left\{(1+d) S_{0}-K, 0\right\}+p_{u}^{*} \max \left\{(1+u) S_{0}-K, 0\right\}\right] \\
P_{0} & =\frac{1}{1+r} E^{*}[G(T)] \\
& =\frac{1}{1+r}\left[p_{d}^{*} \max \left\{K-(1+d) S_{0}, 0\right\}+p_{u}^{*} \max \left\{K-(1+u) S_{0}, 0\right\}\right]
\end{aligned}
$$

We next consider the two-steps binomial tree model defined by

$$
\left\{\begin{array}{l}
P^{*}\left\{S\left(\frac{T}{2}\right)=\left(1+\frac{u}{2}\right) S(0)\right\}=p_{u}^{*} \\
P^{*}\left\{S\left(\frac{T}{2}\right)=\left(1+\frac{d}{2}\right) S(0)\right\}=p_{d}^{*}
\end{array}\right.
$$

and

$$
\left\{\begin{array}{l}
P^{*}\left\{S(T)=\left(1+\frac{u}{2}\right) S\left(\frac{T}{2}\right)\right\}=p_{u}^{*} \\
P^{*}\left\{S(T)=\left(1+\frac{d}{2}\right) S\left(\frac{T}{2}\right)\right\}=p_{d}^{*}
\end{array}\right.
$$

Then we have 


$$
\left\{\begin{array}{l}
P^{*}\left\{S(T)=\left(1+\frac{u}{2}\right)^{2} S(0)\right\}=\left(p_{u}^{*}\right)^{2} \\
P^{*}\left\{S(T)=\left(1+\frac{d}{2}\right)\left(1+\frac{u}{2}\right) S(0)\right\}=2 p_{u}^{*} p_{d}^{*} \\
P^{*}\left\{S(T)=\left(1+\frac{d}{2}\right)^{2} S(0)\right\}=\left(p_{d}^{*}\right)^{2}
\end{array}\right.
$$

\subsection{Option Pricing for Multi-Steps Binomial Model}

We next consider an extension of the multi-steps ( $n T$ steps) binomial tree model defined by

$$
\left\{\begin{array}{l}
P^{*}\left\{S\left(\frac{k}{n}\right)=\left(1+\frac{u}{n}\right) S\left(\frac{k-1}{n}\right)\right\}=p_{u}^{*} \\
P^{*}\left\{S\left(\frac{k}{n}\right)=\left(1+\frac{d}{n}\right) S\left(\frac{k-1}{n}\right)\right\}=p_{d}^{*}
\end{array}\right.
$$

for $k=1,2, \cdots, n T$. Then

$$
P^{*}\left\{S(T)=\left(1+\frac{d}{n}\right)^{k}\left(1+\frac{u}{n}\right)^{n T-k} S_{0}\right\}=\left(\begin{array}{c}
n T \\
k
\end{array}\right)\left(p_{u}^{*}\right)^{k}\left(p_{d}^{*}\right)^{n T-k},
$$

for $k=0,1,2, \cdots, n T$. The call option premium for the multi-steps binomial model is the following

$$
\begin{aligned}
C_{0} & =\frac{1}{\left(1+\frac{r}{n}\right)^{n T}} E^{*}[D(T)] \\
& =\frac{\sum_{k=0}^{n T}\left(p_{d}^{*}\right)^{k}\left(p_{u}^{*}\right)^{n T-k} \max \left\{\left(1+\frac{d}{n}\right)^{k}\left(1+\frac{u}{n}\right)^{n T-k} S(0)-K, 0\right\}}{\left(1+\frac{r}{n}\right)^{n T}}
\end{aligned}
$$

\subsection{Non-Standardization for Option Pricing}

We next consider an extension of the multi-steps ( $n T$ steps) binomial model to the infinitesimal scale model from the view point of the non-standard analysis. The binomial distribution is calculated by the convolution of sums of i.i.d. random variables. Therefore we should apply the notion of the convolution in the non-standard sense.

Consider a stock price defined by the Black-Sholes model

$$
S(t)=S(0) \exp \left(\left(\mu-\frac{\sigma^{2}}{2}\right) t+\sigma W(t)\right), 0 \leq t \leq T .
$$

Then the price of the European call option with a strike price $K$ is given by

$$
V(S, T)=E^{*}[\max \{S(t)-K, 0\}],
$$

where $E^{*}$ is the expectation with respect to the risk neutral probability. To in- 
vestigate the call option pricing Black and Scholes (1973) solve the following PDE

$$
\frac{\partial V}{\partial t}+\frac{1}{2} \sigma^{2} S^{2} \frac{\partial^{2} V}{\partial S^{2}}+r S \frac{\partial V}{\partial S}-r V=0
$$

where $V=V(S, t)$ is the option price at time $t$. This investigation using the standard-analysis is so complicated.

Since it is impossible to calculate $V(S, t)$ with respect to the Wiener measure W directly by the usual standard method, Black and Scholes [1] showed their famous formula for the option pricing to solve the PDE (5).

For a pay-off function $h(s(W))$, the option price with respect to $h(s(W))$ is represented by

$$
V(T)=\int_{C[0, T]} h(s(\omega)) \mathbf{W}(\mathrm{d} \omega)
$$

where for the risk neutral interest rate $r \geq 0$

$$
s(\omega)=\left\{S(0) \exp \left[\left(r-\frac{\sigma^{2}}{2}\right) t+\sigma \omega(t)\right], 0 \leq t \leq T\right\}
$$

Putting $\omega(t)=W(t), s(W)$ is the Black-Scholes model defined by

$$
s(W)=\left\{S(0) \exp \left[\left(r-\frac{\sigma^{2}}{2}\right) t+\sigma W(t)\right], 0 \leq t \leq T\right\} .
$$

A typical example of the pay off function $D(S(W))$ for the strike price $K$ of the European call option is

$$
D(s(W))=\max \left\{S_{T}-K, 0\right\} .
$$

\section{Heat Equations}

Consider a thin circular plate whose faces are impervious to heat flow and whose circular edge is kept zero.

Initial temperature of the plate at $t=0$ is a function $F(r)$ of the distance from the center. Let the radius of the plate be $a$. It is obvious because of symmetry that the temperature $v$ must be a function of $r$ and $t$ only.

Using cylindrical coordinates,

$$
\frac{\partial v}{\partial t}=h^{2} \nabla^{2} v=h^{2}\left(\frac{\partial^{2} v}{\partial r^{2}}+\frac{1}{r} \frac{\partial v}{\partial r}\right), \quad 0<r<a,
$$

where the boundary condition:

$$
v=0, \quad \text { at } t=a
$$

The initial condition:

$$
v=F(r), \quad \text { at } t=0
$$

We easily find that the Black-Sholes Equation (5) can be implied by the Heat Equation (10).

Put $v=\mathrm{e}^{-m t} u(r)$. Then the heat equation is transformed to 


$$
-m u=h^{2}\left(\frac{\partial^{2} v}{\partial r^{2}}+\frac{1}{r} \frac{\partial v}{\partial r}\right)
$$

Furthermore we have

$$
r \frac{\mathrm{d}^{2} u}{\mathrm{~d} r^{2}}+\frac{\mathrm{d} u}{\mathrm{~d} r}+\frac{m}{h^{2}} r u=0,
$$

which is a well-known Bessel equation.

It is known that the solution of the Bessel's equation is described by the first and the second Bessel function. They are typical special functions and described by the Fourier series. The Fourier series is calculated by the Fourier integral. When calculating Fourier integral, the convolution integral is applied. Here, let us consider the extension of the Bessel differential equation to the form in non-standard analysis directly. In fact, changing the standard real valuables to the non-standard valuables in a sense of Nelson. "Transfer Principle" [7] is effective to solve the equation. Then, if the convolution integral is extended to the nonstandard form, see the Section 2, it is sufficient to get the solutions.

\section{Conclusion}

Taking into non-standardization [8] [9] for option pricing, that is, discretizing Wiener process in a sense of the nonstandard, we confirm that it is possible to discretize all the system. If the convolution is extended by using non-standard analysis, and if the hyper functions are satisfied with the conditions in the Section 2, we can obtain the solution for the Black-Sholes equation, which is described as the Fourier integral. Notice that they are of the Bessel special functions.

\section{Conflicts of Interest}

The authors declare no conflicts of interest regarding the publication of this paper.

\section{References}

[1] Black, F. and Scholes, M. (1973) The Pricing of Options and Corporate Liabilities. Journal of Political Economy, 81, 637-654. https://doi.org/10.1086/260062

[2] Kinoshita, M. (1988) Non-Standard Representations of Distributions I. Osaka Journal of Mathematics, 25, 805-824.

[3] Mandelbrot, B.B. and Hudson, R.L. (2004) The (Mis) Behavior of Markets. Basic Books, New York.

[4] Albeverio, S., Fenstad, J.E., Hoegh-Krøhn, R. and Lindstrøm, T. (1986) Nonstandard Methods in Stochastic Analysis and Mathematical Physics. Academic Press, Cambridge.

[5] Benoit, E. (1989) Diffusions discrètes et mécanique stochastique. Prépubli. Université de Nice, Nice.

[6] Benoit, E. (1995) Random Walks and stochastic Differential Equations. In: Diener, F.M., Ed., Nonstandard Analysis in Practice, Springer, Berlin, 71-90. https://doi.org/10.1007/978-3-642-57758-1_4 
[7] Keisler, H.J. (1977) Foundations of Infinitesimal Calculus. Prindle, Weber \& Schmidt, Boston.

[8] Lobry, C. and Sari, T. (2008) Nonstandard Analysis and Representation of Reality. International Journal of Control, 81, 517-534. https://doi.org/10.1080/00207170701601728

[9] Diener, F. and Diener, M. (1995) Tutorial. In: Diener, F.M., Ed., Nonstandard Analysis in Practice, Springer, Berlin, 1-21.

https://doi.org/10.1007/978-3-642-57758-1_1 